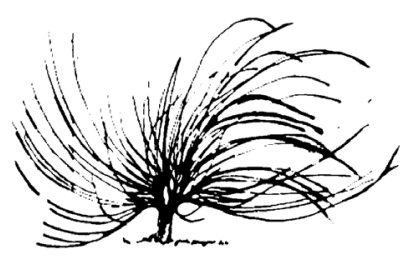

\title{
Análisis de variables relevantes para la promoción del léxico y el desempeño académico en estudiantes de primer año de nivel superior
}

\author{
Martín Gonzalo Zapico ${ }^{1}$ \\ Instituto de Formación Docente Continua San Luis \\ Universidad Nacional de San Luis \\ Argentina \\ athenspierre@gmail.com \\ Lucía Degregori ${ }^{2}$ \\ Universidad Nacional de Mar Del Plata \\ Argentina \\ lucia.degregori@gmail.com \\ Itatí Berardo \\ Universidad Nacional de Mar Del Plata \\ Argentina \\ itaa.blue@gmail.com
}

\begin{abstract}
Resumen
El desempeño académico es un fenómeno multivariable y de complejo análisis. Entre las múltiples causas que se han estudiado, el léxico destaca como una variable interesante en tanto, además de ser explicativa, es una habilidad que puede ser entrenada y reforzada en un tiempo relativamente breve. Dada esta situación, en este trabajo nos propusimos analizar cuáles variables son las más
\end{abstract}

Recibido: 19 de julio de 2019. Aprobado: 29 de abril de 2020.

http://dx.doi.org/10.15359/rep.15-2.5

1 Es Profesor de Letras recibido en la UNMDP. Actualmente se desempeña como Profesor Responsable en el IFDC-San Luis y Doctorando en Educación en la UNSL. Ha realizado numerosas publicaciones en revistas científicas, así como tres libros y asistido a numerosas reuniones científicas en los campos de educación, lingüística y psicología.

2 Es Profesora de Inglés recibida en la UNMDP.

3 Es Profesora de Inglés recibida en la UNMDP. 
importantes en términos de una puntuación alta en un test de léxico. Se analizó edad, sexo, escuela de procedencia, escolarización máxima alcanzada por los padres y hábitos de lectura en 200 ingresantes de distintas carreras de la Universidad Nacional de Mar de Plata y la Universidad Nacional de San Luis en el año 2019. Los resultados indican que la variable que tiene una mayor incidencia en términos estadísticos es la de hábitos de lectura, lo cual significa que es necesario promover esta práctica desde los niveles primario y secundario.

Palabras clave: desempeño académico, educación superior, léxico

\begin{abstract}
Academic performance is a multivariate phenomenon of complex analysis. Among the multiple causes that have been studied, lexicon stands out as an interesting variable since, in addition to being explanatory, it is a skill that can be trained and reinforced in a relatively short time. Given this situation, in this paper we proposed to analyze which variables are the most important in terms of a high score in a lexical test. We analyzed age, sex, school origin, maximum schooling reached parents and reading habits in 200 freshmen from different majors at Universidad Nacional de Mar de Plata, Argentina and Universidad Nacional de San Luis, Argentina in the year 2019. The results indicate that the variable that has the greatest incidence in statistical terms is that of reading habits, which means that it is necessary to promote this practice from primary and secondary levels.
\end{abstract}

Keywords: academic performance, lexicon, higher education

\title{
1. Introducción
}

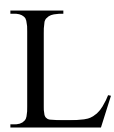

a educación superior refiere al último nivel de formación educativa que se desarrolla en la mayoría de los países del mundo en el ámbito institucional de las universidades e institutos de formación profesional. Clark (1991) lo caracterizará como un sistema conformado por cuatro elementos que, en contraste con otros niveles educativos, se ordenan de forma particular. El primero será una ontología organizada alrededor del conocimiento, que llevará a estructurar prácticas y relaciones entre sus individuos diferenciadas de 
otros ámbitos sociales e incluso de otros niveles del sistema educativo. El segundo es el trabajo que ordena a los profesionales, fundamentalmente, en torno a dos conceptos bien diferenciados, la disciplina y el lugar de trabajo, que a su vez darán lugar a distintas jerarquizaciones explícitas e implícitas que no existen, o no existen de forma tan marcada en, por ejemplo, los niveles primario y secundario. El tercero son las creencias que se constituyen tanto desde el nivel superior e inciden sobre la actividad en forma directa, como aquellas que se construyen desde otros niveles $u$ otros lugares sociales y condicionan el quehacer en este. Y por último la autoridad, que, a diferencia de otros niveles educativos, tiende a distribuirse a lo largo de diferentes modalidades de acuerdo con los distintos contextos de la educación superior.

Este panorama, basado en el análisis de la educación superior mexicana, es fácilmente extrapolable al contexto latinoamericano en general y al argentino en particular. López (2008) señaló que, pese a las diferencias geográficas y culturales, los sistemas de educación superior en América Latina y el Caribe tienen una gran cantidad de parecidos en torno a su organización social y funcionamiento. Una idea similar habría sido expresada tiempo atrás por Altbach (2001), que mostraría como hay un mayor parecido entre los sistemas de educación superior de países disímiles geográfica y hasta culturalmente, que entre los sistemas de educación secundaria y superior de un mismo país.

Es precisamente por este factor que varios autores (Fernández y Carlino, 2010; Otero y Cérica, 2017; Bocchio y Miranda, 2018) han señalado que se producen brechas a veces insalvables entre los últimos años del nivel secundario y el primer año de la educación superior. Como consecuencia se ha generado un problema que ya está naturalizado en los circuitos de este sistema: el abandono universitario. Este fenómeno (Zapico, 2019), entendido como la falta de continuidad definitiva de un sujeto en las instituciones de nivel superior, ha sido reportado numerosas veces, tanto en comunicaciones formales, como artículos y libros (Barsky, Sigal y Dávila, 2012; Giovagnoli, 2001), así como en seminarios, ponencias e incluso charlas cotidianas con gran cantidad de docentes de distintas instituciones, tanto en universidades nacionales como institutos de formación docente.

En este marco, muchos estudios (Armenta, Pacheco y Pineda, 2008; Tejedor, Gonzáles y Señorian, 2008; Vázquez, 2012 y Serrano Ruiz, 2013, entre otros) se han interesado en la búsqueda de variables 
que permitan explicar un desempeño satisfactorio en el primer año del nivel mencionado, puesto que una gran cantidad del abandono en los primeros años está vinculado a la falta de capacidades léxicas (Zapico y Delsouc, 2017, Zapico y Zapico, 2018; Zapico 2018) o vinculadas a la lectoescritura (Carlino, 2001; Carlino, 2007; Carlino, 2013; Carlino, 2017) a la hora de afrontar tareas como trabajos prácticos, exámenes escritos, orales, entre otros. En términos de las variables que garantizan un buen desempeño, hay dos que destacan por su eficacia: factores motivacionales y el nivel socioeconómico. El problema con estos factores es que, en general, las instituciones tienen poca o ninguna capacidad de control sobre ellas, e incluso cuando las tienen, las formas de abordaje son de naturaleza longitudinal, lo que dificulta el sostenimiento de estudiantes en riesgo de abandono.

Por este motivo, resulta valioso el análisis del léxico (Zapico, 2018) y su incidencia en el desempeño académico, porque de perfilarse como una variable significativa (y parece que lo es, por los estudios mencionados anteriormente), es posible la búsqueda de programas de alfabetización específicos para reforzar este aspecto. Así, en este trabajo proponemos hacer un recorrido distinto. En vez de analizar cómo el léxico productivo incide en el desempeño académico, factor que ha sido bastante puesto a prueba con éxito, vamos a analizar cuáles son las variables a priori que llevan a los sujetos a tener un léxico sólido. Dado que no se registran en español estudios donde se analicen qué variables incidan de forma directa en el léxico productivo, nos centraremos en variables más bien generales tales como: sexo, edad, tipo de escuela secundaria, máximo nivel de escolarización de padres y hábitos de lectura. El objetivo es observar cuáles de las variables que resultan significativas pueden ser trabajadas por instituciones de nivel superior o en su defecto en niveles anteriores, y poder separarlas de aquellas que están fuera de alcance institucional. Esta nueva dimensión es re-pensar la problemática del abandono, puesto que, por ejemplo, sabemos que la falta de escolarización máxima alcanzada por los padres suele ser una variable que predice el abandono; sin embargo, si esta no se correlaciona con el desempeño en el test de léxico, podemos enfatizar en la importancia de este y su promoción para favorecer la continuidad de los sujetos en el sistema de forma indirecta.

En este marco, nos propusimos analizar cuáles son las variables que inciden en el léxico. Para dicho propósito, indagamos en las 
puntuaciones de léxico de doscientos sujetos que ingresaban al nivel superior, y observamos las posibles correlaciones entre dichas puntuaciones y otras variables generales.

\section{Metodología}

De acuerdo con Sabino (2014), el estudio se enmarca dentro de las investigaciones aplicadas de carácter cuasiexperimental, dado que se busca relevar una serie de características empíricas y analizarlas para obtener conclusiones sobre la muestra elegida, con un grado de manipulación de variables bajo o casi nulo. A su vez, en términos de la profundización del objeto de estudio, es una investigación del tipo descriptiva-correlacional, pues se busca observar la relación entre el léxico productivo y otras variables. Finalmente, en términos de los tipos de datos empleados, se trata de una investigación cuantitativa.

La investigación constó de dos momentos diferentes. En un primer momento, se recabaron los datos demográficos y de variables de contraste para los sujetos, y se aplicó un test de léxico productivo para determinar el grado de disponibilidad léxica. En un segundo momento, se realizó la estadística descriptiva y correlacional entre las variables de interés y la puntuación en el test de léxico.

\subsection{Instrumento y materiales}

Se utilizó la prueba de vocabulario productivo Z-Test de sinónimos, para cuya elaboración se tomaron las Normas de Producción de Atributos Semánticos en Castellano Rioplatense (Vivas, Comesaña, García Coni, Vivas y Yerro, 2013), a partir de las cuales se obtuvieron 100 palabras de alta frecuencia que poseen sinónimos, según WordReference diccionario de sinónimos (2016). Luego, a través de la comparación con la base CREA de las 1000 palabras más frecuentes de la Real Academia Española (2016) y el juicio de pares especialistas en el área empleando el método de agregados individuales (5 expertos), se llegó a una lista final de 40 palabras, de la que solo se tuvo en cuenta el $100 \%$ de coincidencia favorable, para una mayor solidez del instrumento. Una vez obtenidos estos 40 ítems, se realizó una prueba de IVC con un valor de $.99, \mathrm{~N}=5 \mathrm{y} \mathrm{Ne}=5$, al ser expertos diferentes a los empleados en la etapa anterior del proceso y obtener una puntuación de 1 . Para la validación externa, se realizó una prueba piloto $\mathrm{N}=32$, en la que se contrastaron los resultados obtenidos en el Z-Test con los derivados 
de evaluaciones formales del sistema de educación. Dado que hay un amplio acuerdo entre la relación del vocabulario de amplio espectro con el desempeño académico, puesto que en este se ven involucradas tareas de lectura y escritura, se esperaba observar correlaciones entre ambos resultados. El resultado de la prueba ANOVA, con un p.crítico $=0.05$ y $\mathrm{F}=2.63$, fue de $\mathrm{p}=0.04$, lo que muestra una relación sólida entre las variables mencionadas.

Para el cálculo del coeficiente de validez interna, se contrastaron las puntuaciones obtenidas en la prueba piloto $\mathrm{N}=124$ con las logradas por los mismos sujetos en el Test de denominación de Boston (Serrano et al. 2001). Tras aplicar una estadística de correlación lineal de Pearson, se obtuvo $r=0.78$, que es significativamente alto, si se considera que el test mencionado mide el vocabulario en sus dos dimensiones. Si a esto le sumamos la controversia histórica y actual sobre la necesidad o no de dividir dichas dimensiones, nos encontramos ante una validez interna que es al menos suficiente. Dada la naturaleza de lo que se mide, el vocabulario, se descartó la posibilidad de utilizar un método de test-retest, puesto que había alto riesgo de que los sujetos desarrollaran aprendizaje. Por ello, se aplicó la técnica conocida como formas paralelas, en la que se emplearon 2 versiones equivalentes del Z-Test, con una distancia de una hora entre ambas; se encontró un $\mathrm{r}$ pearson $=$ 0.86 , lo cual indica que prácticamente el $90 \%$ de la varianza del test no se debe al error. En este test, las puntuaciones obtenibles pueden ir desde 0 a 40 y se delimitan 4 grupos de forma orientativa: A ( $>30$ alta competencia léxica), $\mathrm{B}(20<30$ buena competencia léxica $), \mathrm{C}(10<20$ regular competencia léxica) y $\mathrm{D}(<10$ baja competencia).

Para los datos de las otras variables, se empleó una planilla simple con ítems simples para contestar del tipo sexo (M/F), edad (en años), escuela secundaria procedencia (pública/privada), máximo nivel escolarización de los padres (Primaria, secundaria, superior, posgrado), hábitos de lectura (Baja lectura: leyó ningún libro completo el último año, media: leyó 1-2 libros completos el último año, alta: leyó al menos 3 libros completos el año pasado; escala adaptada de Pérez y Barberis (2005). Además, para el procesamiento y análisis de los datos se emplearon los software End Note y el Statistic Package for Social Sciences (SPSS). 


\subsection{Muestra}

El muestreo empleado fue probabilístico, mediante una técnica de aleatorio simple entre la población de ingresantes a distintas carreras de la Universidad Nacional de Mar del Plata. Se obtuvo N $=200$ con un $64.50 \%$ mujeres (129) y $35.50 \%$ hombres (71); de entre 18 y 30 años $(\mathrm{x}=20.77 ; \mathrm{s}=3.80 ; \mathrm{s} 2=14,47)$; de los cuales $56.50 \%$ eran de escuelas públicas (113) y $43.50 \%$ eran de escuelas privadas (87); con al menos uno de los padres con nivel primario completo $11.50 \%$ (23), secundario completo $55.50 \%$ (111), superior completo $19,50 \%$ (39) y posgrado completo $13,50 \%$ (27); con hábitos de lectura distribuidos en $41.00 \%$ bajo (82), $44.50 \%$ medio (89), $14.50 \%$ alto (29).

\subsection{Procedimiento}

Una vez obtenido el consentimiento oral que fue posteriormente respaldado por su versión escrita, se procedió a la administración del Z-Test junto con la planilla para la recolección de información de cada sujeto. Estos se llevaron a cabo de manera individual en el espacio de trabajo del docente-investigador, bajo condiciones óptimas de iluminación y sonido, sin distractor alguno. El tiempo de administración fue de 30 minutos, aunque ningún sujeto superó dicha medida.

\subsection{Análisis}

Una vez obtenidas las puntuaciones de los test, se realizaron los estadísticos descriptivos de tendencia central (media, mediana, moda) junto con sus correspondientes medidas de dispersión (desviación típica, estándar y varianza) y se graficaron las puntuaciones a partir de las distintas variables propuestas. Asimismo, se aplicaron pruebas de normalidad para determinar las características de las puntuaciones obtenidas, junto con los test de homocedasticidad para la determinación del tipo de estadísticos a emplear (paramétricos o no paramétricos de acuerdo con el caso). Realizadas dichas pruebas, se llevaron a cabo los estadísticos de correlación (Anova para los paramétricos y K-Wallis para los no paramétricos) entre la variable léxico y las otras variables. Identificadas variables significativas, se construyó un Modelo Lineal General Multivariante para determinar el peso relativo de cada una de las variables más destacadas. 


\section{Resultados y discusión}

\subsection{Puntuaciones en el test}

En primer lugar, vamos a revisar las puntuaciones en los test de forma amplia, sin tener en cuenta ninguna de las variables en estudio.

\section{Tabla 1}

Estadísticos descriptivos para puntuaciones del test de toda la muestra

\begin{tabular}{|l|c|c|c|c|c|c|c|c|c|}
\hline & N & Mínimo & Máximo & Media & $\begin{array}{c}\text { Desviación } \\
\text { estándar }\end{array}$ & \multicolumn{2}{|c|}{ Asimetría } & \multicolumn{2}{|c|}{ Curtosis } \\
\hline & Valor & Valor & Valor & Valor & Valor & Valor & $\begin{array}{c}\text { Error } \\
\text { estándar }\end{array}$ & Valor & $\begin{array}{c}\text { Error } \\
\text { estándar }\end{array}$ \\
\hline Puntuación & 200 & 7.00 & 38.00 & 19.3300 & 6.97527 & .470 & .172 & -.432 & .342 \\
\hline $\begin{array}{l}\text { N válido } \\
\text { (por lista) }\end{array}$ & 200 & & & & & & & & \\
\hline
\end{tabular}

Nota: Tabla elaborada por los autores

Como se ve en la Tabla 1, la media de las puntuaciones obtenidas es de 19.33, siendo un máximo de puntuación posible 40. Este valor de media es consistente con los observados en puntuaciones obtenidas por otras cohortes de primer año, para distintas carreras en distintas instituciones (Zapico, 2018; Zapico y Delsouc, 2017; Zapico y Zapico 2018; Zapico, Degregori y Berardo, en prensa).

Lo que en primera instancia puede parecer un desempeño aceptable, debe ser matizado por el hecho de que el test es sensible a puntuaciones bajas, es decir, es fácil saturar puntuaciones altas, lo cual con una desviación estándar de casi 7 puntos, habla de una gran cantidad de estudiantes que llegan con un léxico bastante bajo y los que llegan a puntuaciones más altas no pasan el mínimo de éxito establecido en Zapico y Delsouc (2017) donde se analizó una puntuación mínima en la cual los sujetos lograban desempeñarse de manera aceptable a lo largo de un año lectivo, con 25 puntos.

Esto tiende a redundar en problemas para la lectura y comprensión de los textos académicos fundamentales (Bidiña, Kleiman, Lacalle y Toledo, 2018), ya ni hablar de las exigencias de la escritura académica que requiere un esfuerzo de léxico productivo alto. Esta idea de bajas puntuaciones se ve reforzada por la idea de una distribución asimétrica hacia la izquierda (0.47), con una curtosis suave $(-0.43)$, que indica que la mayor parte de las puntuaciones se sitúa en el costado bajo de 
la distribución normal y homocedástica. Los valores mínimos y máximos (7 y 38 respectivamente) que podrían parecer alentadores, se ven aminorados cuando se observa que las puntuaciones altas, en realidad, son casos anómalos, fenómeno observable en la Figura 1 (Box-Plot de distribución de los datos analizados).

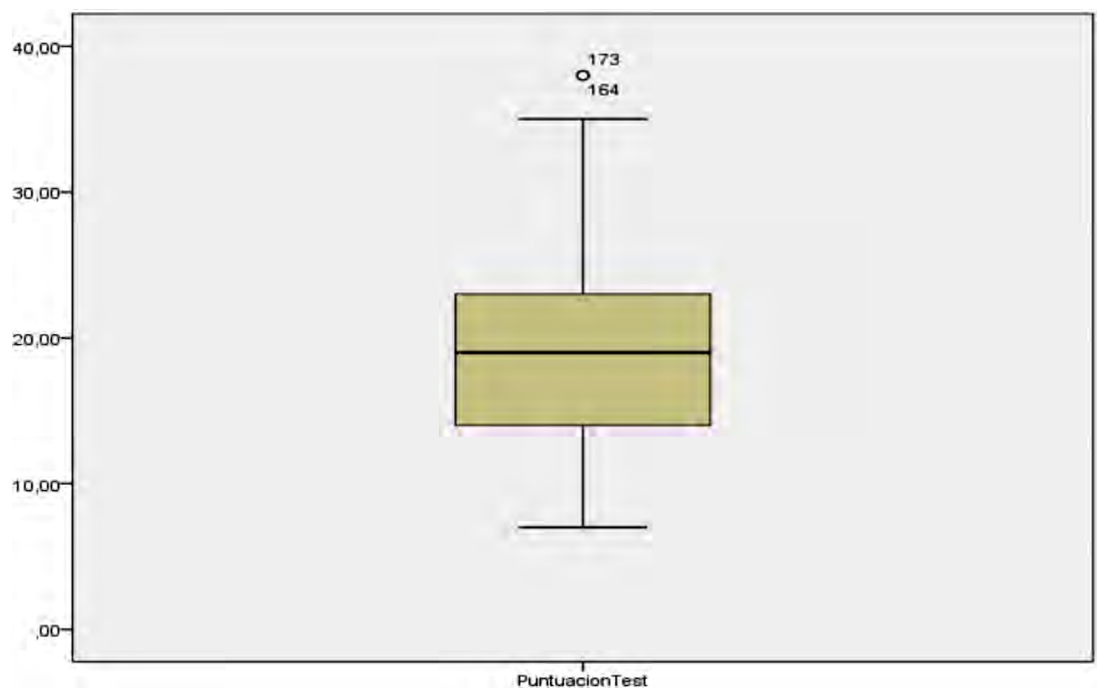

Figura 1. Gráfico de Box-Plot de la distribución de puntuaciones

Nota: Elaboración de los autores

En la Figura 1, se aprecia lo descrito anteriormente, y se suma una nueva medida de tendencia central que refuerza nuestra lectura previa, la Mediana $=19$ que sitúa al $50 \%$ de los valores por debajo de ella y al otro $50 \%$ de los valores por arriba. Como último detalle, hay que señalar que se trata de una distribución con Moda $=20$, que consolida una concentración de puntuaciones en un número por debajo de lo que se espera tenga un estudiante de primer año para tener un buen desempeño.

\subsection{Puntuaciones y variables de agrupación}

Ahora bien, cabe preguntarse, ¿cómo se distribuyen estas puntuaciones a partir del análisis de las variables mencionadas en este estudio? Vamos a ver los análisis de Tablas Cruzadas para las puntuaciones en el test y las variables sexo, escuela de origen, escolarización de padres y hábitos de lectura. Para la variable edad, puesto que se trata de una 
variable numérica, se diseñó una regresión lineal con la Prueba X2 de Pearson con un valor $\mathrm{X} 2=-0.082$ y un p.valor $=0.246$ para la regresión lineal, lo que indica una ausencia total de correlación entre ambas variables, razón por la cual no aparece en la tabla.

Tabla 2

Medidas de centralidad y dispersión en el test por variables de interés

\begin{tabular}{|l|l|c|c|c|c|}
\hline \multicolumn{1}{|c|}{ Variable } & Variante & Media & Mediana & Dest & Min/Max \\
\hline Sexo & Hombre & 18.4 & 17 & 7 & 9 y 34 \\
\hline & Mujer & 19.8 & 20 & 7 & 7 y 38 \\
\hline E. origen & Pública & 18.7 & 18 & 7 & 7 y 38 \\
\hline & Privada & 20 & 20 & 7 & 7 y 38 \\
\hline MGEPadres & Primaria & 17.5 & 17 & 7.2 & 7 y 31 \\
\hline & Secundaria & 20 & 20 & 7 & 7 y 38 \\
\hline & Superior & 18 & 16 & 7 & 8 y 38 \\
\hline & Posgrado & 20.4 & 20 & 6.4 & 9 y 33 \\
\hline & Bajo & 15.8 & 16 & 5.5 & 7 y 32 \\
\hline & Medjo & 20 & 20 & 6 & 9 y 38 \\
\hline & Alto & 27 & 28 & 6 & 14 y 38 \\
\hline
\end{tabular}

Nota: Elaboración de los autores

En la Tabla 2, respecto a la variable sexo, se reafirma lo observado en estudios anteriores y en gran cantidad de estudios sobre léxico (Ávila, 1988; Jiménez, González y Vega, 2018), las mujeres suelen tener mejores desempeños en gran cantidad de habilidades vinculadas a lo lingüístico, y lo leve de estas diferencias en este caso (menos del 10 $\%$ ), vuelven poco interesante a este factor. No obstante, es destacable también observar un fenómeno que se repite vinculado a habilidades lingüísticas, así como las mujeres suelen tener una media siempre más alta que los hombres, también son las poseedoras de las puntuaciones más altas y más bajas, lo que define un rango mucho más amplio, así como una mediana marcadamente superior. No obstante, estas diferencias suelen estar atribuidas a factores biológicos y sociales (Cerdán, 2016), que difícilmente la escuela podrá trabajar, en pos de que los hombres mejoren sus puntuaciones.

Enfocándonos ahora en la variable escuela de origen, encontramos una pequeña diferencia (poco más del $10 \%$ ), que se refleja en las 
puntuaciones de Media y Mediana, donde los estudiantes provenientes de escuelas privadas tienen puntuaciones apenas mayores. Sin embargo, dadas las medidas de dispersión tan similares, así como los puntajes mínimos y máximos, no es una diferencia que sea ni estadísticamente testeable, ni tampoco trabajable desde un punto de vista institucional. Para una discusión sobre las diferencias entre escuelas públicas y privadas en Argentina véase Orellana, Caviedes, Belloi y Contreras (2018), donde se analiza el fenómeno desde un punto de vista social y vinculado a la elección de instituciones de nivel superior. Aquí se refleja que, si bien es cierto los estudiantes provenientes de escuelas privadas tienen ventajas en términos de formación respecto a los de escuelas públicas, muchas de esas ventajas están más vinculadas a la variable socioeconómica de la familia que a la formación de la escuela en sí.

Muy vinculada a la variable anterior, encontramos la de máximo grado de escolarización de los padres que, en este caso, parece no tener una incidencia importante en las puntuaciones. Ni los hijos de padres con una escolarización máxima de primaria tienen puntuaciones pésimas, ni aquellos hijos de padres con nivel de posgrado tienen puntuaciones altas. Si bien es cierto que entre estos dos grupos hay una diferencia considerable, las dos categorías del medio parecen mezclarse entre sí sin ningún tipo de correlación observable. Lo que es interesante destacar es cómo los extremos de escolarización parecen definir dos grupos bien diferentes de estudiantes, notorias tanto en la media, la mediana, la D. Estándar y los Puntajes Min/Max. Si bien muchos estudios relacionan esta variable con el desempeño académico, esto suele estar fuertemente vinculado a los niveles de educación primario y secundario, pues dicha influencia ya desaparece en el nivel superior donde los sujetos gozan de una mayor autonomía respecto a la familia y han atravesado doce años de escolarización.

La última variable es la de hábitos de lectura. Esta, ya a simple vista parece tener una incidencia importante en la distribución de las puntuaciones del test. Tanto la media como la mediana aumentan significativamente, y a medida que esto ocurre las puntuaciones mínimas y máximas también aumentan. En este punto, parece evidente que esta variable es fuerte en términos de explicar las puntuaciones en el test. Sin embargo, es necesario probar si estas diferencias son estadísticamente significativas. 


\subsection{Hábitos de lectura}

Para dicho propósito, primero establecemos las pruebas de normalidad (Kolgomorov-Smirnov, con un p.valor $=0.2)$ y de homocedasticidad (Test de Levene, con un p.valor $=0.73$ ) que confirman una distribución normal y homocedástica. Esto nos da la pauta de que se debe realizar un ANOVA Unifactorial, con la variable dependiente puntuación en el test y la dependiente Hábitos de lectura, con los resultados presentados a continuación en la Tabla 3.

\section{Tabla 3}

Prueba ANOVA con variable dependiente test e independiente hábitos de lectura

\begin{tabular}{|l|c|c|c|c|c|}
\hline & $\begin{array}{c}\text { Suma de } \\
\text { cuadrados }\end{array}$ & gl & $\begin{array}{c}\text { Media } \\
\text { cuadrática }\end{array}$ & F & Sig. \\
\hline Entre grupos & 2941.018 & 2 & 1470.509 & 42.973 & .000 \\
\hline Dentro de grupos & 6741.202 & 197 & 34.219 & & \\
\hline Total & 9682.220 & 199 & & & \\
\hline
\end{tabular}

Nota: Elaboración de los autores

Con un p.valor $=0,000$ confirmamos que hay una correlación estadísticamente significativa entre las variables mencionadas. Esta relación reviste un grado de interés alto no solo por el valor de la prueba, sino porque la variable hábitos de lectura es una variable sobre la cual distintos organismos de educación en distintos niveles pueden tener incidencia y estimular una práctica más recurrente. Al analizar más detenidamente las correlaciones con una Tabla de Comparaciones Múltiples con un Estadístico sensible como DMS y uno algo más rígido como Tukey, encontramos también una correlación alta para todas las variantes de la variable. 
Análisis de variables relevantes para la promoción del léxico y el desempeño académico en estudiantes de primer año de nivel superior

Tabla 4

Comparaciones múltiples para hábitos de lectura

\begin{tabular}{|c|c|c|c|c|c|c|c|}
\hline & $\begin{array}{c}\text { (I) } \\
\text { HLectura }\end{array}$ & $\begin{array}{c}(\mathrm{J}) \\
\text { HLectura }\end{array}$ & $\begin{array}{c}\text { Diferencia de } \\
\text { medias (I-J) }\end{array}$ & $\begin{array}{c}\text { Error } \\
\text { estándar }\end{array}$ & Sig. & & \\
\hline & & & & & & $\begin{array}{l}\text { Límite } \\
\text { inferior }\end{array}$ & \\
\hline \multirow[t]{6}{*}{ HSD Tukey } & Bajo & Medio & $-4.17361^{*}$ & .89543 & .000 & -6.2882 & -2.0590 \\
\hline & & Alto & $-11.58663^{*}$ & 1.26384 & .000 & -14.5713 & -8.6020 \\
\hline & Medio & Bajo & $4.17361^{*}$ & .89543 & .000 & 2.0590 & 6.2882 \\
\hline & & Alto & $-7.41302^{*}$ & 1.25078 & .000 & -10.3668 & -4.4592 \\
\hline & Alto & Bajo & $11.58663^{*}$ & 1.26384 & .000 & 8.6020 & 14.5713 \\
\hline & & Medio & $7.41302^{*}$ & 1.25078 & .000 & 4.4592 & 10.3668 \\
\hline \multirow[t]{6}{*}{ DMS } & Bajo & Medio & $-4.17361^{*}$ & .89543 & .000 & -5.9395 & -2.4078 \\
\hline & & Alto & $-11.58663^{*}$ & 1.26384 & .000 & -14.0790 & -9.0942 \\
\hline & Medio & Bajo & $4.17361^{*}$ & .89543 & .000 & 2.4078 & 5.9395 \\
\hline & & Alto & $-7.41302^{*}$ & 1.25078 & .000 & -9.8797 & -4.9464 \\
\hline & Alto & Bajo & $11.58663^{*}$ & 1.26384 & .000 & 9.0942 & 14.0790 \\
\hline & & Medio & $7.41302^{*}$ & 1.25078 & .000 & 4.9464 & 9.8797 \\
\hline
\end{tabular}

Nota: Elaboración de los autores

Lo atractivo de la Tabla 4 y sus resultados tan pronunciados es que señalan que cada salto en términos de hábitos de lectura representa una mejora en las puntuaciones de léxico productivo. Es decir que hay una relación al parecer directa entre el acto de leer y la consolidación no solo de un aspecto pasivo del léxico, sino también del activo. Esto resulta de especial interés, pues si esta tendencia se confirma en futuros estudios, traería nuevamente a la escena las hipótesis vinculados a la exposición directa para adquisición de vocabulario.

Al tener esto como punto de partida, se buscó luego la construcción de un Modelo Lineal General, para lo cual se realizaron pruebas de Chi-Cuadrado entre la variable Hábitos de lectura y las demás variables (se salva la de edad para la cual se realizó un K-Wallis), se obtuvieron los siguientes resultados (en todos los casos con un nivel de significación de 0.05). 


\section{Tabla 5}

Pruebas entre hábitos de lectura y otras variables

\begin{tabular}{|l|c|}
\hline \multicolumn{1}{|c|}{ Variable } & Chi-Cuadrado \\
\hline Sexo & 0.39 \\
\hline E. Origen & 0.88 \\
\hline MGEPAdres & 0.28 \\
\hline & K. Wallis \\
\hline Edad & 0.71 \\
\hline
\end{tabular}

Nota: Elaboración de los autores

El análisis de la Tabla 5 pone de manifiesto la imposibilidad de construir un modelo más complejo, puesto que con estos valores cualquier tipo de análisis multivariado no añadirá más información nueva. Sin embargo, se puede observar que hay un grado de asociación (estadísticamente insignificante puesto que es un p.valor no significativo, pero cualitativamente interesante porque pone de manifiesto la primacía de la lectura como una práctica) entre la variable de hábitos de lectura y el máximo grado de escolarización de los padres. Es decir, de manera indirecta, se puede afirmar que la estimulación a la lectura parece estar dada fundamentalmente por la influencia de los padres antes que por el tipo de escuela de procedencia, la edad o el sexo. Esto puede ser tomado como punto de partida por instituciones de todos los niveles a la hora de optar por tender canales de comunicación entre la escuela (ya sea primaria o secundaria) y los grupos familiares.

Asimismo, y al retomar lo analizado sobre las puntuaciones, se realizaron las pruebas de Anova o K-Wallis (según correspondiese), para descartar posibles correlaciones, se observa resultados nada significativos para cada uno de los casos (Edad p.valor $=0.10$, Sexo p.valor $=0.08$, E. Procedencia p.valor $=0.16$, MGEscolarización Padres p.valor $=0.12$; en todos los casos con un nivel de significación de 0.05). Es decir, de todas las variables mencionadas en la Tabla 2, la única que se aproximó a un resultado estadísticamente significativo fue sexo, lo cual era en alguna medida esperado de acuerdo a estudios previos. 


\section{Reflexiones}

Hemos tenido la oportunidad de analizar algunas de las variables asociadas al rendimiento académico a través de sus vinculaciones con las puntuaciones en un test de léxico. De este análisis, ha surgido la importancia de los hábitos de lectura como variable explicativa en términos de las puntuaciones obtenidas. Es decir, el desempeño académico ha sido previamente correlacionado, de forma exitosa, con el léxico productivo, y a su vez, este parece estar fuertemente correlacionado con los hábitos de lectura. Esto es importante, puesto que la lectura y su habitualidad es algo que las instituciones tanto de nivel superior, como en los niveles primario y secundario, pueden afectar mediante políticas específicas de difusión y promoción de la lectura, que no necesariamente debe ser académica sino que puede ser también lúdica.

A su vez, hemos observado también que dichos hábitos de lectura se relacionan de manera débil pero vinculante con el nivel de escolarización alcanzado por los padres. Esto puede interpretarse como la contracara del esfuerzo institucional a la hora de buscar una mayor habituación a las prácticas lectoras: la promoción de la lectura desde el ámbito familiar, allí donde muchas veces la institución no tiene potestad ni poder de acción, también es un espacio importante para consolidar las habilidades léxicas de los estudiantes.

Como limitantes a este estudio es necesario señalar que el $\mathrm{N}$ es relativamente bajo, la muestra se limitó a una sola Universidad Nacional. En próximos estudios se buscará ampliar la muestra, para incluir institutos de formación docente y universidades privadas, se sumará la variable riqueza léxico, así como la incorporación de factores de control como diagnóstico de TEL o Afasias, que podrían incidir negativamente en el desempeño en el test.

Finalmente, hay que destacar la importancia de la promoción de la enseñanza activa del léxico en los distintos niveles de la educación formal, asunto ya analizado en estudios anteriores, como una vía de combatir el creciente analfabetismo académico al que, tanto docentes como alumnos, deben enfrentar en sus prácticas diarias. De esta forma, no solo estaremos dando herramientas para la vida académica, sino también para la vida en sociedad, puesto que no hay actividad alguna que no sea mediada por el lenguaje. 


\section{Referencias}

Altbach, P. G. (2001). Educación superior comparada. Ciudad de Buenos Aires: Universidad Palermo.

Armenta, N., Pacheco, C. y Pineda, E. (2008). Factores Socio-Económicos que intervienen en el desempeño académico de los estudiantes universitarios de la facultad de ciencias humanas de la Universidad Autónoma de Baja California. Revista IIPSI, 11 (1), 153-165.

Ávila, R. (1988). Lenguaje hablada y estrato social: un acercamiento lexicoestadístico. Nueva Revista de Filología Hispánica, Madrid, XXXVI (1), 131-148.

Barsky, O., Sigal, V. y Dávila, M. (2012). Los desafios de la universidad argentina. Buenos Aires: Siglo XXI.

Bidiña, A., Kleiman, F. G., Lacalle, J. M., y Toledo, V. (2018). La mediación de las TIC en el ingreso a la comunidad académica: una primera experiencia en el Seminario de Comprensión y Producción de Textos del ingreso a la Universidad Nacional de La Matanza. Rihumso: Revista de Investigación del Departamento de Humanidades y Ciencias Sociales, 13, 115-134.

Bocchio, M. C. y Miranda, E. (2018). La escolaridad secundaria obligatoria en Argentina. Política para la inclusión socio-educativa en la escuela. Revista Educación, 1(1), 769-793.

Carlino, P. (2017). Dos variantes de la alfabetización académica cuando se entrelazan la lectura y la escritura en las materias. Signo y Pensamiento, 36(71), 18-34.

Carlino, P. (2013). Alfabetización académica diez años después. Revista mexicana de investigación educativa, 18(57), 355-381.

Carlino, P. (2007). ¿Qué nos dicen las investigaciones internacionales sobre escritura en la universidad? Cuadernos de Psicopedagogía, 4(1), 21-40.

Carlino, P. (2001). Hacerse cargo de la lectura y la escritura en la enseñanza universitaria de las ciencias sociales y humanas. I Jornada sobre la lectura y la escritura como prácticas académicas universitarias. Departamento de Educación, Universidad Nacional de Luján, Luján, Prov. de Buenos Aires.

Cerdán, A. (2016). Estudio de la respuesta del electromiograma ante distintos estímulos: efectos del sexo y características del estímulo 
Análisis de variables relevantes para la promoción del léxico y el desempeño académico en estudiantes de primer año de nivel superior

(Tesis para aplicar al grado de licenciatura). Universidad Miguel Hernández, Elche.

Clark, B. (1991). El sistema de educación superior. Una visión comparativa de la organización académica, Universidad Autónoma Metropolitana-Azcapotzalco. Ciudad de México: Editorial Nueva Imagen Universidad Futura.

Fernández, G., y Carlino, P. (2010). ¿En qué se diferencian las prácticas de lectura y escritura de la universidad y las de la escuela secundaria? Lectura y vida, 31(3), 6-19.

Giovagnoli, P. (2001) Determinantes de deserción y graduación universitaria (Tesis para aplicar al grado de licenciatura). Universidad de La Plata, Ciudad de La Plata.

Jiménez, Á. G., González, S. G., y Vega, G. T. (2018). Disponibilidad léxica de hablantes extranjeros estudiantes de español como segunda lengua en Santiago de Chile: Estudio preliminar de las diferencias de sexo. Káñina, 42(2), 231-256.

López, F. (2008). Tendencias de la educación superior en el mundo y en América Latina y el Caribe. Avaliação: Revista da Avaliação da Educação Superior (Campinas), 13(2), 267-291.

Orellana, V., Caviedes, S., Bellei, C. y Contreras, M. (2018). La elección de escuela como fenómeno sociológico. Una revisión de literatura. Revista Brasileira de Educação, 23, 134-149.

Otero, A., y Cérica, A. (2017). La performance del sistema educativo argentino en las últimas décadas. Análisis. Revista Colombiana de Humanidades, 49(90), 17-37.

Pérez, V. M. O., y Barberis, L. T. (2005). Análisis de los hábitos de estudio en una muestra de alumnos universitarios. Revista Iberoamericana de Educación, 36(7), 1-9.

Sabino, C. (2014). El proceso de investigación. Buenos Aires: Editorial Episteme.

Serrano, C., Allegri, R. F., Drake, M., Butman, J., Harris, P., Nagle, C., y Ranalli, C. (2001). Versión abreviada en español del test de denominación de Boston: su utilidad en el diagnóstico diferencial de la enfermedad de Alzheimer. Revista de neurología, 33(7), 624-627.

Serrano, L (2013). La influencia de los factores socio-económicos en el rendimiento académico (Tesis para aplicar al grado de máster). Universidad de Almería, Almería. 
Tejedor, F., González, S. y Señorian, M. (2008). Estrategias atencionales y rendimiento académico en estudiantes de secundaria. Revista Latinoamericana de Psicología, 40 (1), 5-15.

Vázquez, C. (2012). Factores de impacto en el rendimiento académico universitario. Un estudio a partir de las percepciones de los estudiantes. Decimoséptimas Jornadas "Investigaciones en la Facultad" de Ciencias Económicas y Estadística. Universidad Nacional de Rosario, Provincia de Santa Fé.

Vivas, J., Comesaña, A., García Coni, A., Vivas, L., y Yerro, M. (2013). Distribución de los atributos semánticos en función del tipo de categoría y campo semántico. Resultados preliminares para la confección de normas de atributos. En M. Richaud y V. Lemos (Ed.), Psicología y otras ciencias del comportamiento. Compendio de investigaciones actuales I (pp. 311-333). Libertador San Martin: Editorial Universidad Adventista del Plata.

Zapico, G. (2019). ¿Deserción Universitaria? Una reflexión conceptual en torno a la problemática. Analéctica, 5 (34), 1-8.

Zapico, G. (2018). Adquisición de vocabulario productivo en contextos académicos superiores: análisis de su evolución a partir de un estudio longitudinal. Revista Ensayos Pedagógicos 13(2), 119-137.

Zapico, G., Degregori, L. y Berardo, I. (En prensa). ¿Hay una disminución en la cantidad de vocabulario de los ingresantes a carreras de nivel superior? Análisis longitudinal. Revista Ensayos Pedagógicos.

Zapico, G y Delsouc, A (2017). Competencia léxico-productiva, memoria a corto plazo y desempeño académico en el nivel superior: correlaciones a partir de un análisis simple y uno multivariado. Revista Ensayos Pedagógicos, 12(1), 149-165.

Zapico, G y Zapico, G (2018). La competencia léxico-productiva como predictor del desempeño académico. Revista Ensayos Pedagógicos, 13(1), 223-243. 